\title{
Comparison of the Linear Matching Method to Rolls-Royce's Hierarchical Finite Element Framework for ratchet limit analysis
}

\author{
Michael Lytwyn ${ }^{\mathrm{a}}$, Haofeng Chen ${ }^{\mathrm{a} *}$, Michael Martin ${ }^{\mathrm{b}}$ \\ ${ }^{a}$ Department of Mechanical \& Aerospace Engineering, University of Strathclyde, Glasgow, G1 1XJ, UK \\ ${ }^{b}$ Rolls-Royce Power Engineering plc., PO Box 2000, Derby, DE21 7XX, UK
}

\begin{abstract}
This paper provides a direct comparison between the Linear Matching Method (LMM) and the numerical procedures currently being employed within the Rolls-Royce Power Engineering (plc) Hierarchical Finite Element Framework (HFEF) for the assessment of shakedown and ratcheting behaviour. These numerical methods include the application of Direct Cyclic Analysis (DCA), utilised in an automated search procedure for load-interaction plot generation and the recently developed Hybrid procedure. The Hybrid procedure is based on a similar premise to the LMM in that the load history is decomposed into cyclic and constant components. The LMM allows for the direct evaluation of shakedown and ratchet limits to be obtained in a traditional Bree loadinteraction format, along with the subsequent maximum plastic strain range for low-cycle fatigue considerations. Three problems have been used for comparison in this paper; the classic Bree cylinder, a nozzle-in-sphere with a cold media injection transient typical of nuclear power plant loading and a pressurised two-bar structure for multi-axial failure analysis. The accuracy of each method has been verified using ABAQUS step-by-step inelastic analysis. The variations in the implementation strategies associated with each method have also been discussed along with computational efficiency and effectiveness, which show that the LMM has the significant potential to improve analysis speeds via obtaining the ratchet limit boundary directly for a specified level of cyclic loading, instead of conducting an iterative search procedure.
\end{abstract}

Keywords: ratchet limit; shakedown; linear matching method; direct cyclic analysis.

*Corresponding author - Email: haofeng.chen@strath.ac.uk 


\begin{tabular}{|ll|}
\hline Nomenclature & \\
$P(x, t)$ & Cyclic component of load history (mechanical load), at integration point $x$ and time \\
$\theta(x, t)$ & point $t$ during the cycle. \\
$\lambda$ & Cyclic component of load history (thermal) \\
$\mathrm{n}$ & Load scaling factor \\
$\mathrm{N}$ & Load instance number \\
$\tilde{\sigma}_{i j}$ & The total number of time points in the cycle \\
$\sigma_{i j}(x, t)$ & Stress tensor \\
$\tilde{\sigma}_{i j}$ & Linear elastic stress history \\
$\tilde{\sigma}_{i j}^{\Delta}(x, t)$ & Vlastic stress associated with the additional constant load component \\
$\sigma_{p}$ & Mechanical load component \\
$\bar{\rho}_{i j}$ & The constant component of the changing residual stress \\
$\rho_{i j}^{r}(x, t)$ & The varying residual stress field \\
$\mathrm{t}_{\mathrm{n}}$ & Time point within the cycle \\
$\rho_{i j}\left(t_{n}\right)$ & The accumulated residual stress at load instance $\mathrm{t}_{\mathrm{n}}$ \\
$\Delta \varepsilon_{i j}^{n}$ & The increment of plastic strain at load instance $\mathrm{n}$ \\
$\bar{\varepsilon}$ & Von Mises effective strain \\
$\sigma_{y}$ & \\
\hline
\end{tabular}

\section{Introduction}

In the nuclear industry, structural integrity assessments are undertaken to the requirements of recognised international standards such as ASME III to provide through-life assurance against the occurrence of potential structural failure modes, including ductile burst, ratcheting and fatigue. This paper is concerned with the prediction of shakedown and prevention of the ratcheting failure mode. Ratcheting is associated with structures operating at temperature and pressure, whereby under 
certain cyclic load conditions the structure accrues a net increment of plastic strain with each application of the load cycle, thus eventually leading to failure. Under certain circumstances, the load history may be such that the accumulated plastic strains cease to develop after a few initial load cycles, known as elastic shakedown.

Rolls-Royce Power Engineering plc. is currently developing the use of 'modern' finite element methods for the assessment of pressure vessel structures to the strength, shakedown and fatigue requirements of ASME III Code, Subsection NB [1]. This is encapsulated in the Hierarchical Finite Element Framework [2], or HFEF. HFEF is based on the application of Limit Load Analysis (LLA), direct shakedown prediction and nodal strain-based fatigue post-processing in lieu of the SCL and linearisation techniques traditionally used to demonstrate acceptance to the primary and secondary stress limits of ASME III Subsection NB. LLA provides assessment of the burst failure mode whilst direct shakedown prediction and strain-based fatigue analysis respectively assess the incremental collapse and fatigue failure modes. The HFEF methods do not require the application of stress classification lines (SCLs) or their associated stress classification and linearization procedures. If the stable cyclic strain range includes a fluctuating plastic component without a net accumulation after each cycle, then reversed or alternating plasticity is observed.

Recent shakedown analysis methods have been developed by applying plasticity bounding theorems $[3,4]$ in tandem with modern FEA packages and mathematical optimization theory. Such methods are known as direct shakedown analysis methods and offer the advantage that the exact load history is not required for implementation purposes, only the most significant loads acting on the structure need to be specified and the shakedown theorems applied to determine a safe operational envelope in load space. The recent advances in direct shakedown approaches has led to a significant reduction in analysis times for the assessment of progressive plastic deformation in components subjected to high temperature operating conditions without sacrificing accuracy, compared to the traditional step-by-step FE methods commonly used for verification purposes. Such step-by-step methods require significant computation time for ratchet boundary prediction and cannot predict a ratchet limit directly, thus only being capable of indicating if elastic shakedown, plastic shakedown or ratcheting occur at a certain point in the load domain. Direct methods can be 
seen to offer a supporting alternative or complete replacement of the traditional (SCL) methods, until modern computer facilities advance enough to allow step-by-step methods to become considerably more efficient. Examples of such direct methods include the Linear Matching Method [5, 6, 7], the Nonlinear Superposition Method [8], Mathematical Programming Methods [9] and Repeated Elastic Analysis methods; including Seshadri's GLOSS r-node method [10]. The LMM is distinguished from other direct methods by ensuring that equilibrium and compatibility are satisfied at each stage of analysis as well as the ability to incorporate high temperature material behaviour [6].

The LMM has recently been developed to incorporate multi-load extremes in the thermomechanical load domain [7] and as such offers a robust and accurate method for obtaining the ratchet boundary in a direct manner. The LMM process involves calculating the load carrying capacity of a structure subjected to a predefined cyclic load component ensued by the addition of an extra constant load in order to determine the proximity of the ratchet limit. This methodology allows the stable cyclic response, i.e. the cyclic stress, residual stress and plastic strain ranges for the low-cycle fatigue assessment to be computed [7]. The entire LMM numerical procedure for both shakedown and ratcheting assessment has been incorporated into the commercial finite element code ABAQUS [11]. As a result, the LMM can be readily used for the ratchet limit assessment of structures involving load histories with complex multi-load extremes, as are commonly prevalent in the nuclear industry. A significant advantage of the LMM includes offering pressure vessel designers the ability to assess 3D structures under complex loading and allow for rapid specification of the ratchet load without requiring unrealistic computing facilities as well as ease of user implementation. Within Rolls-Royce's HFEF, two methods which are currently being developed for ratchet boundary prediction include the use of Direct Cyclic Analysis [12, 13] and the Hybrid Procedure, both of which will be briefly summarised in Section 2.1.

This paper aims to compare the LMM with the application of two of Rolls-Royce's HFEF shakedown assessment methods in, Direct Cyclic Analysis (DCA) [13], as well as the recently developed Hybrid procedure [14]. The three example problems that will be used for comparison in this paper include; the classic Bree cylinder that forms the basis of the ASME III shakedown 
assessment $[1,15]$, a nozzle-in-sphere with a cold-media injection transient and a pressurised twobar structure for multi-axial failure considerations, which has been modified from Abdalla's original problem [16] via the inclusion of internal pressure. The comparison results used in this paper for the pure DCA and Hybrid methods have been obtained from [14], in order to form the benchmark data for the models analysed.

\section{An overview of numerical methods for ratcheting analysis}

\subsection{Current numerical procedures for ratchet analysis being developed within Rolls-Royce}

As mentioned, the results which have been used for comparison purposes in this paper have been derived from state of the art numerical ratchet analysis methods which are currently being employed within Rolls-Royce's HFEF. These methods are currently under development and aim to remove the uncertainties associated with the traditional ASME III shakedown assessments, which rely on traditional stress classification procedures, which can often be subjective when implemented in practical plant scenarios. Within HFEF, two methods currently which are currently being developed for ratchet boundary prediction include the use of Direct Cyclic Analysis [13] and the Hybrid procedure [14], these will be briefly explained in this section, however more in-depth details are available in $[2,13,14]$.

DCA is a Fourier based approach which was initially implemented in ABAQUS to detect if a stabilised cyclic response existed and therefore indicate if ratcheting or elastic/plastic shakedown occurred, without determining a ratchet boundary directly $[13,14]$. This method however has been automated in [13] such that a ratchet boundary can be obtained via an optimisation procedure generated using Python [17], whereby varying load levels and the application of several load cycles are used to establish the ratchet boundary. The process involves using repeated DCA calculations and assessing the convergence of the solution in order to indicate if a steady cyclic response has been obtained for a particular load cycle. A bisection algorithm is used to alter the levels of loading for each DCA calculation and to search for the ratchet limit, however in order to differentiate between the strict and global shakedown limits further examination of the plastic strains must be conducted. Due to this search procedure, this method may prove to be time consuming and impractical in an industrial context for problems involving complex thermo-mechanical load 
histories, especially for acute thermal transients, as full details of the load cycle must be considered as well. An example of the application of this search procedure for the Bree problem and the resulting series of individual DCA calculations can be seen in Fig. 1 (from the work of [13]).

The Hybrid methodology stems from recent work undertaken by Reinhardt [18] and Adibi-Asl et al [19]. The fundamental concept of the Hybrid method is to ascertain the steady cyclic response of a structure undergoing cyclic loading using DCA in a first stage, followed by an intermediate step where a unit vector of steady cyclic loading is added to each extreme in the load cycle. This unit vector of steady cyclic stress can then be scaled to the yield surface for each extreme of the load cycle, in order to ascertain the level of the level of constant loading that may be applied to each respective load extreme. The minimum value of additional constant stress obtained from scaling this vector can then be taken as the effective yield stress for a second stage calculation (this process is implemented at each integration point in the model in order for modified yield stress values to be obtained for the entire volume). The second stage then involves conducting a limit load calculation based upon using the remaining yield capacity of the structure (using the modified yield strength values from stage one of the method), in order to obtain the ratchet boundary with respect to an additional constant loading. It is worth noting that any cyclic solution procedure be actually be used to calculate the modified yield strength values from the steady cyclic response of the problem.

Both the application of pure DCA and the Hybrid methodology demonstrated in [13] and [14] require the entire cyclic load history to be analysed (at some stage of the analysis), which accounts for larger analysis times compared to the LMM, due to insignificant loads in the cycle being taken into consideration even though they may not contribute to the overall global ratcheting failure mechanism. It is noted that some form of cycle simplification may be employed to the DCA and Hybrid methods to filter insignificant cycles and this is an ongoing area of research.

\subsection{The Linear Matching Method}

The evolution of the LMM has evolved from using Koiter's upper bound (UB) kinematic theorem [3] initially in terms of limit analysis [20] and then shakedown analysis [21], before the extension of the upper bound (and Melan's lower bound theorem) was proposed for the purpose of 
more advanced ratcheting analysis and the assessment of problems involving varying residual stress fields by Ponter, Chen et al [7, 22, 23, 24].

From a continuum mechanics viewpoint, we can consider a body with volume $\mathrm{V}$ and surface $\mathrm{S}$, where the material is isotropic, elastic-plastic and satisfies the von Mises yield condition. A cyclic temperature field $\theta(x, t)$ occurs within the volume V. A cyclic mechanical load $P(x, t)$ is applied over part of the surface $\mathrm{S}$, namely $\mathrm{S}_{\mathrm{T}}$. The notation " $P(x, t)$ " refers to the location in the volume $(x)$ and time point during the cycle $(t)$ (with $x$ representing an integration point in the context of a typical FE model). Here $\lambda$ denotes a scalar load parameter. On the remainder of $\mathrm{S}$, namely $\mathrm{S}_{\mathrm{u}}$, zero displacements are maintained. Both $\theta(x, t)$ and $P(x, t)$ act over a cycle $\Delta t$, with $0 \leq t \leq \Delta t$. The complete history of applied cyclic loads $F(x, t)$ applied to the volume may be shown as:

$$
F(x, t)=\theta(x, t)+P(x, t)+\lambda \bar{F}(x)
$$

Where $\lambda \bar{F}(x)$ represents an additional constant mechanical load (i.e. load controlled) which may be scaled using the load factor $\lambda$. The LMM ratchet analysis calculation requires the applied loads in Eq. (1) to be decomposed into separate cyclic and constant components, i.e. in order to initially assess the combined effect of the cyclic loads $\theta(x, t)+P(x, t)$ in terms of obtaining the steady cycle behaviour, before moving on to calculate the amount of additional constant loading $\lambda \bar{F}(x)$ that the structure may accommodate before ratcheting occurs in a subsequent calculation; thus a two stage numerical procedure is employed. The reason for doing so is to enable separate calculations of the varying and constant residual stress fields associated with the cyclic and constant loads to be conducted respectively.

In order to expand upon this point further, the general form of the cyclic stress solution, relative to the applied loads from Eq. (1), may be seen as;

$$
\sigma_{i j}(x, t)=\lambda \tilde{\sigma}_{i j}^{\bar{F}}+\tilde{\sigma}_{i j}^{\Delta}(x, t)+\rho_{i j}^{r}(x, t)+\bar{\rho}_{i j}(x)
$$

Where $\tilde{\sigma}_{i j}^{\Delta}(x, t)$ is the stress associated with the combined action of the cyclic thermal and 
mechanical loads $\theta(x, t)+P(x, t)$ and $\tilde{\sigma}_{i j}^{\bar{F}}$ is the additional constant stress caused by $\bar{F}(x)$. The term $\bar{\rho}_{i j}(x)$ denotes a constant residual stress field which is in equilibrium with zero surface traction on $\mathrm{S}_{\mathrm{T}}$ and corresponds to the residual state of stress at the beginning and end of each cycle, whilst $\rho_{i j}^{r}(x, t)$ represents any changes of residual stress during each cycle due to cyclic plasticity and can be seen to remain constant from one cycle to the next upon reaching a stable cyclic period, i.e.

$$
\rho_{i j}^{r}(x, 0)=\rho_{i j}^{r}(x, \Delta t)=\bar{\rho}_{i j}^{r}(x)
$$

Where $\bar{\rho}_{i j}^{r}(x)$ is the constant component of the varying residual stress, which will be expanded upon in Section 2.2.1. Eqs. (1-3) presented thus far are in a general format, in terms of describing the cyclic plasticity problem presented in this paper, irrespective of any detailed numerical discussion, i.e. the magnitudes all refer to converged quantities in the context of a numerical scheme. Further details of such numerical considerations shall be presented in the following sections.

\subsubsection{Stage 1: The iterative LMM procedure for the evaluation of the varying residual stress history associated with a predefined cyclic load history}

As mentioned, a two stage procedure is employed in order to calculate the ratchet limit using the LMM. Stage 1 involves an incremental minimisation process for the evaluation of the cyclic history of varying residual stresses $\rho_{i j}^{r}(x, t)$ and subsequent plastic strain range in a stable cycle, caused by the predefined cyclic load history $\tilde{\sigma}_{i j}^{\Delta}(x, t)$. In order to evaluate the varying residual stress history caused by this predefined cyclic load range, a series of elastic solutions are initially generated at various discrete time points in order to effectively sample the entire load cycle (for example at $n$ locations in time, so that a subsequent series of elastic solutions are generated, $\tilde{\sigma}_{i j}^{\Delta}\left(x, t_{n}\right)$ (where $n=1, \ldots N$ total number of load instances). The important assumption that any cyclic plasticity will only occur at the load extremes $n=1, \ldots N$ is then stipulated.

In the context of a numerical procedure, Stage 1 of the LMM ratchet analysis requires a series of 
iterative cycles, ranging from $m=1, \ldots, M$ cycles (upon reaching convergence), with each cycle containing a series of $N$ increments; relative to the amount of discrete elastic solutions adopted as an input to the problem. In the first iteration of the method for the first iterative cycle (e.g. $n=1$ and $m$ $=1$ ), we evaluate the first increment of the varying residual stress $\Delta \rho_{i j}^{r}\left(x, t_{1}\right)_{m}$ which is attributed to the elastic solution at the first load instance $\tilde{\sigma}_{i j}^{\Delta}\left(x, t_{1}\right)$, i.e. the varying residual stress at a particular load instance $n$ and cycle number $m$ is defined as $\Delta \rho_{i j}^{r}\left(x, t_{n}\right)_{m}$. Thus, the primary emphasis of the LMM Stage 1 procedure is to continue in this manner; by iteratively calculating each individual varying residual stress $\Delta \rho_{i j}^{r}\left(x, t_{n}\right)_{m}$ associated with each elastic solution respectively, from $n=1, \ldots N$, until convergence is reached at cycle $M$.

During each iterative cycle, the accumulated varying residual stress for each load instance is iteratively updated by taking into account magnitudes from previous cycles, combined with the values obtained from the current iteration of the method for that particular load instance, i.e;

$$
\rho_{i j}^{r}\left(x, t_{n}\right)_{m}=\sum_{k=1}^{m-1} \sum_{n=1}^{N} \rho_{i j}^{r}\left(x, t_{n}\right)_{k}+\sum_{l=1}^{n} \rho_{i j}^{r}\left(x, t_{l}\right)_{m}
$$

Upon reaching convergence at the Mth cycle of iterations, the summation of the varying residual stresses across $\mathrm{N}$ load instances will approach to zero, due to stable cyclic conditions (i.e. $\bar{\rho}_{i j}^{r}(x)$ from Eq. (3)), such that;

$$
\sum_{n=1}^{N} \Delta \rho_{i j}^{r}\left(x, t_{n}\right)_{M}=0
$$

Once the solution has converged, we can then expand the constant component of the varying residual stress $\bar{\rho}_{i j}^{r}(x)$ into the form;

$$
\bar{\rho}_{i j}^{r}(x)=\sum_{m=1}^{M} \sum_{n=1}^{N} \rho_{i j}^{r}\left(x, t_{n}\right)_{m}
$$


The final value of accumulated varying residual stress attained upon convergence can be seen in Eq, (7), which combines the accumulated (constant) residual stress $\bar{\rho}_{i j}^{r}(x)$ from Eq. (6) and takes into account the varying residual stresses from the final iterative cycle $M$ across each load instance $n$.

$$
\rho_{i j}^{r}\left(x, t_{n}\right)=\bar{\rho}_{i j}^{r}(x)+\sum_{k=1}^{n} \Delta \rho_{i j}^{r}\left(x, t_{n}\right)_{M}
$$

If we have a value of the varying residual stress at cycle $m$ for load instance $t_{n}$, i.e. $\rho_{i j}^{r}\left(x, t_{n}\right)_{m}$, calculated from a previous iteration, we can then utilise a linear matching relationship in order to iteratively calculate each individual varying residual stress (i.e. by means of repetitive elastic calculations). The method utilises the relationship shown in Eq. (8) in order to calculate a linear matching modulus $\mu\left(t_{n}\right)_{m}$;

$$
\mu\left(t_{n}\right)_{m}=\mu\left(t_{n}\right)_{m-1} \frac{\sigma_{y}}{\bar{\sigma}\left(\widetilde{\sigma}_{i j}^{\Delta}\left(x, t_{n}\right)+\rho_{i j}^{r}\left(x, t_{n}\right)_{m}\right)}
$$

Where $\mu\left(t_{n}\right)_{m}$ is iterative matching modulus and $\mu\left(t_{n}\right)_{m-1}$ is the modulus from the previous cycle. Convergence for Stage 1 is adjudged to have been met when a predefined tolerance on the variation of iterative shear moduli from one cycle to the next is satisfied.

\subsubsection{Stage 2: Evaluation of the upper bound ratchet limit}

The second stage of the numerical process involves a global minimization procedure in order to obtain the ratchet limit due with respect to an additional constant load $\lambda \tilde{\sigma}_{i j}^{\bar{F}}$, by means of a modified shakedown analysis; whereby a constant residual stress $\bar{\rho}_{i j}(x)$ is evaluated by utilising the varying residual stress history $\rho_{i j}^{r}(x, t)$ from Stage 1 , in combination with the fixed cyclic load range $\tilde{\sigma}_{i j}^{\Delta}(x, t)$.

The upper bound LMM formulation is derived from Koiter's kinematic theorem [3], which states that if any kinematically admissible strain rate can be located such that the strain field is compatible 
with the applied displacements and that the subsequent plastic dissipation within the problem domain is less than or equal to the applied work, then shakedown does not occur. As described, once the history of the varying residual stresses $\rho_{i j}^{r}(x, t)$ associated with the cyclic component $\tilde{\sigma}_{i j}^{\Delta}(x, t)$ of the load history has been calculated, the numerical technique for the ratchet limit can be accommodated within the existing methods of shakedown analysis; e.g. from using $\left(\tilde{\sigma}_{i j}^{\Delta}(x, t)+\right.$ $\left.\rho_{i j}^{r}(x, t)\right)$ in order to obtain the magnitude of additional constant load $\tilde{\sigma}_{i j}^{\bar{F}}$ that the structure can accommodate before ratcheting occurs. For a von Mises yield condition and the associated flow rule, an upper bound on the ratchet limit multiplier can subsequently be obtained using;

$$
\lambda_{U B}^{R}=\frac{\int_{V} \sum_{n=1}^{N} \sigma_{y} \bar{\varepsilon}\left(\Delta \varepsilon_{i j}^{n}\right) d V-\int_{V} \sum_{n=1}^{N}\left(\sigma_{i j}^{\Delta}\left(x, t_{n}\right)+\rho_{i j}^{r}\left(x, t_{n}\right)\right) \Delta \varepsilon_{i j}^{n} d V}{\int_{V} \sigma_{i j}^{\bar{F}}\left(\sum_{n=1}^{N} \Delta \varepsilon_{i j}^{n}\right) d V}
$$

Eq. (9) produces a sequence of monotonically reducing upper bounds $\lambda_{U B}^{R}$ which converge to the least upper bound ratchet limit for the chosen class of displacement fields. Note the absence of $\bar{\rho}(x)$ in Eq. (9) (i.e. the constant residual stress from Eq. (2)), which may be eliminated due to the application of the virtual work principle [22, 23]. All of the LMM results and numerical considerations in this paper are discussed with respect to the upper bound LMM ratchet analysis framework only. Stages $1 \& 2$ of the LMM have been implemented in ABAQUS via user-defined subroutines (UMAT \& URDFIL), which can accommodate multi-load extremes and temperaturedependent yield stress data. A more comprehensive description of the two stage LMM ratchet assessment may be found in [7].

\section{Bree Cylinder Analysis}

In order to generate the load-interaction plot for the Bree cylinder problem, a finite element model was generated as illustrated in Fig. 2. A cyclic thermal load was applied to the inner surface of the cylinder alongside a constant internal pressure. The applied cyclic thermal and constant pressure loading are shown in Fig. 3. Subsequently the problem can be characterised by the two 
load extremes, $\sigma_{p}$ and $\Delta \theta+\theta_{0}$, (where $\theta_{0}$ is the ambient temperature, i.e. $20^{\circ} \mathrm{C}$ in this case), resulting from the pressure and thermal loads respectively. The geometry of the problem consists of a thin closed-end cylinder, with an internal radius of $365 \mathrm{~mm}$ and an external radius of $385 \mathrm{~mm}$. The FE mesh for the Bree cylinder consisted of 10 elements in the thickness direction, which is deemed sufficient to represent this simple temperature gradient. The cyclic thermal load depicted in Fig. 3 was analysed using quadratic DCAX8 heat transfer elements, with the resulting temperature distributions used as an input to the static analyses, which were performed using quadratic CAX8R reduced integration structural elements.

The cylinder was constrained vertically at one end and allowed to expand in-plane at the free end, with a thrust applied to the free end to simulate a closed-end condition. The following temperature independent material properties were used in the analysis: thermal conductivity $=$ $0.035 \mathrm{~W} / \mathrm{mm}^{\circ} \mathrm{C}$, Young's Modulus $=184 \mathrm{GPa}$, Poisson's ratio $=0.3$, coefficient of thermal expansion $=1.335 \mathrm{e}^{-5 \circ} \mathrm{C}^{-1}$ and $\sigma_{y}=402.7 \mathrm{MPa}$. The resulting pressure versus cyclic temperature interaction diagram found using the LMM, Hybrid and pure DCA methods can be seen in Fig. 4 (with the DCA and Hybrid results obtained from separate work in [13] and [14]). In order to generate such a plot using the DCA method alone, the method in [13] implemented an automated bisection algorithm in Python, such that the ratchet boundary was located with a tolerance of around $\pm 0.05 \mathrm{MPa}$.

Fig. 4 illustrates that the Hybrid method marginally under predicts the ratchet boundary with respect to the pure DCA method, particularly so as the temperature increases. This can be attributed to the increase in plastic zone size with respect to increasing cyclic temperature, which highlights the limitations within the vector addition approach adopted in the Hybrid method [14]. The reverse plasticity limit has been located at a maximum cyclic temperature of around $500^{\circ} \mathrm{C}$, which has been verified by an elastic-plastic step-by-step analysis, with further inspection of the resulting magnitudes of plastic strain (Fig. 5).

The discrepancies between the LMM derived ratchet boundary and the pure DCA results may be considered small enough to disregard with respect to further step-by-step analysis verification along this boundary, as such studies have previously been completed for the same exact problem [13]. Also shown in Fig. 4, as a dashed line, is the alternating plasticity (AP) limit obtained via the LMM. 
The plastic strain results in Fig. 5 illustrate the stabilised reverse plasticity mechanism at point $\mathrm{A}:\left(\mathrm{p}=11.2 \mathrm{MPa}, \mathrm{T}=515^{\circ} \mathrm{C}\right)$ after 50 cycles, i.e. exhibiting non-incremental plastic strains, as well as the state of elastic shakedown achieved at point $\mathrm{B}:\left(\mathrm{p}=11.2 \mathrm{MPa}, \mathrm{T}=480^{\circ} \mathrm{C}\right)$.

\section{Nozzle-in-sphere with cold shock transient}

This problem provides a more complex analysis case of the methods presented, by simulating the pressurised cold-shock example of the nozzle-in-sphere model and the resulting high transient thermal gradients. The FE mesh consisted of 5717 elements, with linear heat transfer elements (DCAX4) being used for the thermal analysis and quadratic reduced integration elements (CAX8R) with midside node interpolation for the structural analyses.

The vessel includes a $45^{\circ}$ section which is fixed locally in the direction normal to the cut surface, in order to allow radial movement only. An in-plane constraint was applied to the pipe attachment as well as a thrust due to the internal pressure force being exerted on the vessel, as shown in Fig. 6. Details of the problem geometry can found in Fig. 7. The operational history over one cycle can be seen in Fig. 8, which shows the constant pressure load and cyclic thermal transients with a $20^{\circ} \mathrm{C}$ cold-fluid injection. The thermal response of the model is driven entirely by thermal loading through the application of film coefficients, as detailed in Fig. 9.

The model consists of three varying materials, with the relative material parameters shown Tables $1 \& 2$. In order to generate comparison data with the results available for the Hybrid [14] and pure DCA [13] methods, the most severe cold-injection case of $\mathrm{T}_{\mathrm{c}}=20^{\circ} \mathrm{C}$ is used for the $\mathrm{LMM}$ ratchet boundary calculations. The load points used to obtain the thermal stress extremes are shown in Fig. 8 (labelled $\mathrm{t}_{1}-\mathrm{t}_{6}$ ). The thermal stresses are each calculated by an elastic thermal analysis $a$ priori and subsequently used as inputs to the LMM ratchet boundary calculation. Figs. 10 (i-iii) indicate the locations where the most significant stress magnitudes for the problem are situated (for $\mathrm{T}_{\mathrm{c}}=20^{\circ} \mathrm{C}$ ), with i) $\mathrm{T}_{\mathrm{c}}+10 \mathrm{~s}$, ii) $\mathrm{T}_{\mathrm{c}}+600 \mathrm{~s}$ and iii) the additional constant load component.

The cyclic thermal time-points can be seen to be representative of:

- $\mathrm{t}_{1}-$ Ambient conditions.

- $\mathrm{t}_{2}-$ Steady-state operating conditions at $295^{\circ} \mathrm{C}$ uniform temperature. 
- $\mathrm{t}_{3}-10$ seconds after a cold-media injection of $\mathrm{T}_{\mathrm{c}}=20^{\circ} \mathrm{C}$.

- $\mathrm{t}_{4}-600$ seconds after a cold-media injection of $\mathrm{T}_{\mathrm{c}}=20^{\circ} \mathrm{C}$, i.e. steady-state injection.

- $\mathrm{t}_{5}-10$ seconds after the removal of the cold-media and return to steady-state $\left(295^{\circ} \mathrm{C}\right)$ conditions.

- $\mathrm{t}_{6}-2000$ seconds after the removal of the cold-media.

These salient points $t_{1}-t_{6}$ have been identified in order to encapsulate the most significant thermal stress ranges evident in a $20^{\circ} \mathrm{C}$ cold-injection phase and as such are deemed fully representative of the entire cycle in terms of accurately identifying the ratchet boundary directly via the LMM. The cyclic thermal load history must be represented accurately in terms of the relevant peak stresses involved, such that the most onerous stress ranges dominating the failure response of the structure can be identified successfully. Subsequently, the ratchet limit multiplier can therefore be obtained by analysing the capacity of the vessel to withstand the elastic stress ranges from $t_{1}-t_{6}$ in conjunction with an additional constant pressure load, before ratcheting occurs. Fig. 11 depicts the ratcheting boundaries obtained via Hybrid and pure DCA methods respectively for comparison purposes. The ratchet boundary derived from the LMM assessment can be seen to almost exactly follow the data obtained using the Hybrid method. In order to verify the data obtained, step-by-step calculations were conducted for $\mathrm{T}_{\mathrm{c}}=20^{\circ} \mathrm{C}$ at two locations; $\mathrm{C}=17.1 \mathrm{MPa}$ and $\mathrm{D}=17.5 \mathrm{MPa}$, with 100 cycles adopted as shown in Fig. 12. The results from the elastic-plastic verification analyses are taken from 'location 4' (Fig. 13), using a perfectly-plastic material model. As is evident from Fig. 12, point $\mathrm{C}$ shows alternating plasticity and point $\mathrm{D}$ outside the ratchet boundary (obtained via the LMM and Hybrid methods) displays incremental plastic strain accumulation.

The LMM and Hybrid methods both show that for more complex scenarios involving nonproportional loading (where the thermal actions vary with time and are not in phase with the pressure loading), that the application of pure DCA leads to a conservative ratchet boundary prediction. The implementation strategy adopted in the application of pure DCA for ratchet analyses requires considerable computational expense, as the accuracy of the method accuracy relies upon suitable parameter selection, in terms of Fourier terms. Increasing the number of Fourier coefficients directly influences the analysis time. DCA must incorporate the entire load history as an 
input to the problem, before determining whether or not convergence is achieved based on a userdefined iteration limit. Convergence before the iteration limit is reached is therefore deemed to represent shakedown and failure to converge represents ratcheting, or plastic collapse. As discussed previously, the benefits of using the LMM stem from the requirement that only the stress locations in the cycle which contribute to the most severe stress ranges dominating the failure mechanism need to be included in the ratchet boundary calculations, therefore the entire load history does not need to be incorporated into the analysis. The main factor related to the reduction in computation time arises from the LMM producing the ratchet boundary directly, as opposed to searching for this limit by means of a search algorithm in the case of DCA [13].

The maximum plastic strain range generated by the cyclic thermal transient of $\mathrm{T}_{\mathrm{c}}=20^{\circ} \mathrm{C}$ can be seen in Fig. 14 at 'location 5'. This location was noted by Rauscher et al [25] but deemed insignificant in terms of the overall global shakedown response of the structure due to the limited cross sectional extent, however this would be the low-cycle fatigue crack initiation site, attributed solely to the cyclic thermal loading.

\section{Pressurised two-bar structure}

The final example considered consists of a pressurised two-bar structure which is analysed in order to investigate multi-axial failure effects, as both the Bree cylinder and nozzle-in-sphere problems predominantly exhibit failure mechanisms in the hoop direction only. This problem was initially presented by Abdalla et al [16] and subsequently modified by Martin et al [14] with the addition of internal pressure.

A three-dimensional model was constructed of two hollow cylinders with a total of 4700 continuum elements in the FE mesh. The 3D quadratic heat transfer element DC3D20 was used for the thermal analysis, with the element type then switched to a 3D quadratic reduced-integration element, C3D20R, for the structural analyses. Dimensional and material data for this problem can be found in Table. 3. The cyclic load history is created by cycling only the outer temperature of the $200 \mathrm{~mm}$ bar from $0^{\circ} \mathrm{C}$ to $100^{\circ} \mathrm{C}$, with the smaller $100 \mathrm{~mm}$ bar remaining at a constant $0^{\circ} \mathrm{C}$. The boundary conditions consisted of fixing the bottom ends of each bar in the axial direction, with an in-plane constraint being used to apply a constant axial force to each of the free ends as depicted in 
Fig. 15, in order to allow for tandem movement of the free ends. An internal pressure was also added to the $200 \mathrm{~mm}$ bar so that various ratios of axial force $(\mathrm{F})$ to internal pressure $(\mathrm{p})$ could be generated in order to explore multi-axial failure in the problem. In order to implement the LMM for this particular problem, the cyclic load extremes derived from the thermal stresses associated with $0^{\circ} \mathrm{C}$ and $100^{\circ} \mathrm{C}$ temperature magnitudes are cycled in Stage 1 of the LMM ratchet calculation before proceeding to add the mechanical load component in order to obtain the proximity to the ratchet limit, which in this case consisted of the applied axial force and internal pressure. Therefore in order to generate the load-interaction plot for this particular problem, $\Delta \theta=100^{\circ} \mathrm{C}$ was scaled according to the temperature of interest in the LMM ratchet calculations.

Fig. 16 illustrates the results obtained for each LMM analysis relative to the pure DCA and Hybrid solutions, whereby the ratio of axial force $\mathrm{F}$ to internal pressure $\mathrm{p}$ was altered from 10,15 and 20 respectively. As is evident from the results, the most severe case arrives at $\mathrm{F} / \mathrm{p}=10$, where the hoop failure mechanism causes the lowest limit load of all $\mathrm{F} / \mathrm{p}$ ratios and a reduction in the overall ratchet limit, due to the geometry of the problem. As the ratio of F/p is increased, the severity of the hoop stresses are reduced such that a transition to an axially induced failure mechanism occurs, which is less detrimental in terms of ratcheting behaviour and thus an increase in the ratchet limit and limit load is observed. The LMM calculations closely follow the ratchet boundaries obtained from the application of pure DCA, which is deemed to be more representative of the actual response of the structure compared to the Hybrid method results [14], however the LMM can achieve these results with less computational expense as mentioned previously. The inaccuracy of Hybrid method for this problem may be attributable to errors in the vector addition approach as the extent of plasticity develops [14].

\section{Discussion}

In the present study, the LMM has been used to directly evaluate the ratchet limit for three example problems in order to compare results with those obtained via an automated DCA based procedure and a novel Hybrid procedure. Due to the results presented in this paper consisting of work from separate studies, no direct CPU evaluation times have been provided for comparison between the methods. However, even though no such computational times have been provided, the 
main purpose of the work at hand is to primarily illustrate that the LMM is more economical due to the full cyclic load history only needing to be sampled at certain discrete locations within a typical load cycle, as opposed to pure DCA and the Hybrid method, which both must analyse the full cyclic transient; which means even innocuous load excursions will be unnecessarily analysed. In the case of using DCA alone, a search procedure must also be used to locate the ratchet boundary, which reduces the efficiency of this method.

Both of the comparative methods used in this paper rely upon the DCA method and are therefore influenced by the selection of Fourier terms. More Fourier coefficients are required to resolve severe gradients in load and structural response to ensure sufficient accuracy, at the expense of solution time [13]. A benefit of the LMM is also evident in the ability to immediately identify the maximum plastic strain range attributed to the cyclic load history, which can readily be verified by means of elastic-plastic step-by-step FEA.

Noticeably, the third example presented in this paper has alluded to apparent deficiencies within the vector addition approach of the Hybrid methodology, relative to certain levels of cyclic loading, which has led to further re-development of the method. When the results obtained from each method are actually similar in terms of accuracy, then the merits of each procedure may be emphasised further in terms of user implementation as well as computational efficiency aspects.

\section{Conclusions}

The comparative study presented in this paper has shown that the LMM provides a better alternative approach to the use of pure DCA and the Hybrid method for the purpose of ratchet analysis of pressure vessels subject to complex thermo-mechanical loading, due to the direct nature of the upper bound methodology employed and the potential computational savings from using a series of discrete elastic solutions in order to represent a typical cyclic transient. As such, the LMM provides a promising and robust ratcheting analysis approach for inclusion within the Rolls-Royce Power Engineering plc. HFEF methodology. Further application of the LMM to practical pressure vessel structures is required to build experience in the use of the method. 


\section{Acknowledgements}

The authors gratefully acknowledge Rolls-Royce Power Engineering plc. and the University of Strathclyde for their support during this work.

\section{References}

[1] ASME Boiler and Pressure Vessel Code, Section III, Rules for construction of nuclear power plant components, Div. 1, Subsection NB, class 1 components, 2007.

[2] Martin M, Rawson L, Rice D, A hierarchical finite element framework for the assessment of pressure vessels to the ASME III code. ASME Pressure Vessels and Piping Conference, Bellevue, Washington, Paper No. PVP2010-25097, pp. 125-135, 2010.

[3] Koiter WT, General theorems for elastic plastic solids. Progress in solid mechanics J.N. Sneddon and R. Hill, eds. North Holland, Amsterdam, 1, 1960;167-221.

[4] Melan E, Theorie Statisch Unbestimmter Systeme aus Ideal-Plastischem Bastoff. Sitzungsberichte der Akademie der Wissenschaft. Wien, Abtiia, 1936;145, 195-218.

[5] Chen HF, Lower and Upper Bound Shakedown Analysis of structures With TemperatureDependent Yield Stress. Journal of Pressure Vessel Technology, 2010; 132, 1-8

[6] Chen HF, Ponter A.R.S. Linear Matching Method on the evaluation of plastic and creep behaviours for bodies subjected to cyclic thermal and mechanical loading. International Journal for Numerical Methods in Engineering 2006; 68: 13-32

[7] Chen HF, A Direct Method on the Evaluation of Ratchet Limit. Journal of Pressure Vessel Technology, 132(4), 041202, 2010.

[8] Muscat M, Mackenzie D and Hamilton R. Evaluating shakedown by non-linear static analysis. Computers and Structures 2003; 81: 1727-1737.

[9] Staat M, Heitzer M, LISA a European Project for FEM-based Limit and Shakedown Analysis. Nuclear Engineering and Design 2001; 206: 151-166.

[10] Seshadri R, The generalised local stress strain (GLOSS) analysis - theory and application. Trans ASME, Journal Pressure Vessel Technology 1991;113: 219-227.

[11] ABAQUS 6.10, SIMULIA, 2010 
[12] Nguyen-Tajan TML, Pommer B, Maitournam H, Hourari M, Verger L, Du ZZ, Snyman M, Determination of the stabilized response of a structure undergoing cyclic thermal-mechanical loads by a direct cyclic method. ABAQUS Users' Conference Proceedings, 2003

[13] Martin, M. Application of direct cyclic analysis to the prediction of plastic shakedown of nuclear power plant components. ASME Pressure Vessels and Piping Conference, Chicago, Illinois, Paper No. PVP2008-61067, pp. 265-275, 2008.

[14] Martin M, Rice D. A hybrid procedure for ratchet boundary prediction. ASME Pressure Vessels and Piping Conference, Prague, Czech Republic, Paper No. PVP2009-77474, pp. 8188, 2009.

[15] Bree J, Elasto-plastic behaviour of thin tubes subjected to internal pressure and intermittent heat fluxes with application to fast reactor fuel elements. Journal Strain Analysis, 1967 (2), 226-238.

[16] Abdalla HF, Megahed M.M, Younan MYA, A Simplified Technique for Shakedown Limit Load Determination, Nuclear Engineering and Design, 237(2007) 1231-1240.

[17] Python 2.5, www.python.org

[18] Reinhardt W, Elastic-Plastic Shakedown Assessment of Piping using a Non-Cyclic Method, ASME Pressure Vessels and Piping Conference, San Antonio, Texas, Paper No. PVP200726703, pp. 59-70, 2007.

[19] Adibi-Asl R and Reinhardt W, Elastic Modulus Adjustment Procedure (EMAP) for Shakedown, ASME Pressure Vessels and Piping Conference, Chicago, Illinois, Paper No. PVP2008-61641, pp. 823-832, 2008.

[20] Ponter, A.R.S, Carter, K.F., Limit state solutions, based upon linear solutions with a spatially varying elastic modulus, Comput. Methods Appl. Mech. Engrg. 140 (1997) 237-258.

[21] Ponter, A.R.S, Carter, K.F., Shakedown state simulation techniques based on linear elastic solutions, Comput. Methods Appl. Mech. Engrg. 140 (1997) 259-279.

[22] Ponter A. R. S, Chen H. F, A minimum theorem for cyclic loading in excess of shakedown, with applications to the evaluation of a ratchet limit, European Journal of Mechanics A/Solids 2001; 20 (4) 539-554. 
[23] Chen H. F, Ponter A. R. S, A method for the evaluation of a ratchet limit and the amplitude of plastic strain for bodies subjected to cyclic loading, European Journal of Mechanics A/Solids 2001; 20 (4), 555-572.

[24] Chen H.F., Ure J., Tipping D., Calculation of a lower bound ratchet limit part 1 - Theory, numerical implementation and verification, European Journal of Mechanics - A/Solids, 37, pp. 361-368, 2013.

[25] Rauscher F, Design By Analysis Direct Route for Cases With Pressure and Thermal Action, ASME Pressure Vessels and Piping Conference, Vancouver, BC, Canada, Paper No. PVP2006-ICPVT-11-94027, pp. 799-806, 2006. 
Table 1

Table. 1 - Temperature dependent yield strength data (MPa).

\begin{tabular}{|c|cccccc|}
\hline Temperature $\left({ }^{\circ} \mathbf{C}\right)$ & $\mathbf{2 0}$ & $\mathbf{1 0 0}$ & $\mathbf{1 5 0}$ & $\mathbf{2 0 0}$ & $\mathbf{2 5 0}$ & $\mathbf{3 0 0}$ \\
\hline Material 1 & 290 & 249 & 238 & 232 & 227 & 221 \\
& & & & & & \\
Material 2 & 355 & 323 & 312 & 304 & 296 & 289 \\
Material 3 & 265 & 226 & 213 & 192 & 171 & 154 \\
\hline
\end{tabular}


Table 2

Table. 2 - Temperature independent material data.

\begin{tabular}{|c|c|c|c|}
\hline \multirow{2}{*}{ Property } & \multicolumn{3}{|c|}{ Material } \\
\cline { 2 - 4 } & $\mathbf{1}$ & $\mathbf{2}$ & $\mathbf{3}$ \\
\hline Conductivity $\left(\mathrm{W} / \mathrm{mm}^{\circ} \mathrm{C}\right)$ & 0.0349 & 0.0349 & 0.051 \\
Young's Modulus $(\mathrm{GPa})$ & 212 & 212 & 212 \\
Poisson's ratio & 0.3 & 0.3 & 0.3 \\
Expansion $\left({ }^{\circ} \mathrm{C}^{-1}\right)$ & $1.15 \mathrm{e}-5$ & $1.15 \mathrm{e}-5$ & $1.19 \mathrm{e}-5$ \\
Specific heat $\left(\mathrm{J}^{\mathrm{k}} \mathrm{kg}^{\circ} \mathrm{C}\right)$ & 461 & 461 & 461 \\
\hline
\end{tabular}


Table 3

Table. 3 - Pressurised Two-Bar problem material properties.

\begin{tabular}{|l|cc|}
\hline \multicolumn{1}{|c|}{ Property } & Bar 1 & Bar 2 \\
\hline Length $(\mathrm{mm})$ & 100 & 200 \\
Conductivity $\left(\mathrm{W} / \mathrm{mm}^{\circ} \mathrm{C}\right)$ & 0.035 & 0.035 \\
Outside radius $(\mathrm{mm})$ & 2.68 & 3.22 \\
Inside radius $(\mathrm{mm})$ & 2.00 & 2.00 \\
Young's Modulus $(\mathrm{GPa})$ & 210 & 210 \\
Poisson's ratio & 0.3 & 0.3 \\
Expansion $\left({ }^{\circ} \mathrm{C}^{-1}\right)$ & $11.7 \mathrm{e}-6$ & $11.7 \mathrm{e}-6$ \\
Yield Strength $(\mathrm{MPa})$ & 200 & 200 \\
\hline
\end{tabular}




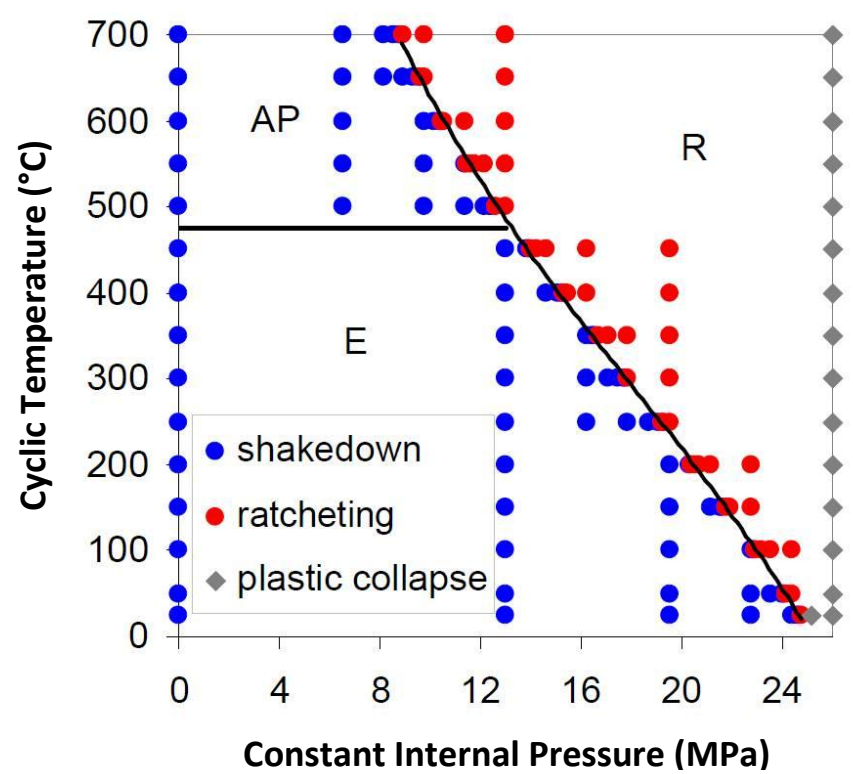

Fig. 1 - Example of DCA trial and error calculations for the classic Bree problem (from [13]). 


\section{Figure 2}

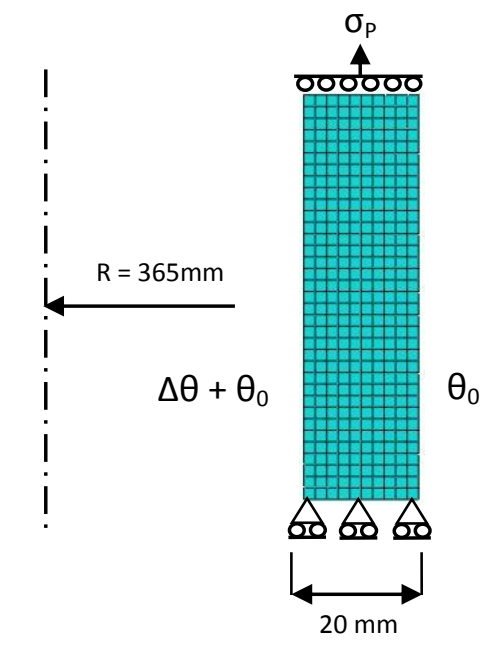

Fig. 2 - Model geometry and axisymmetric FE mesh with boundary conditions. 
Figure 3

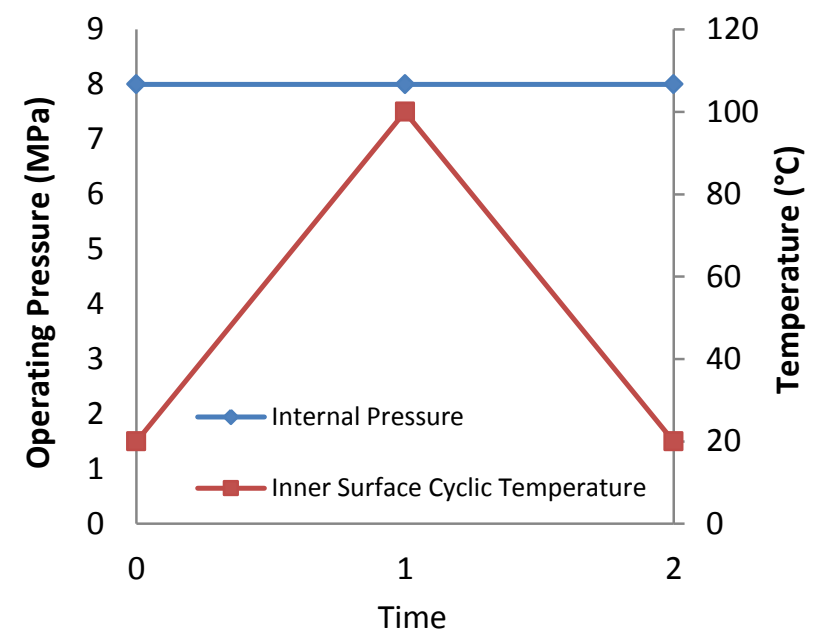

Fig. 3 - Bree cylinder loading. 
Figure 4

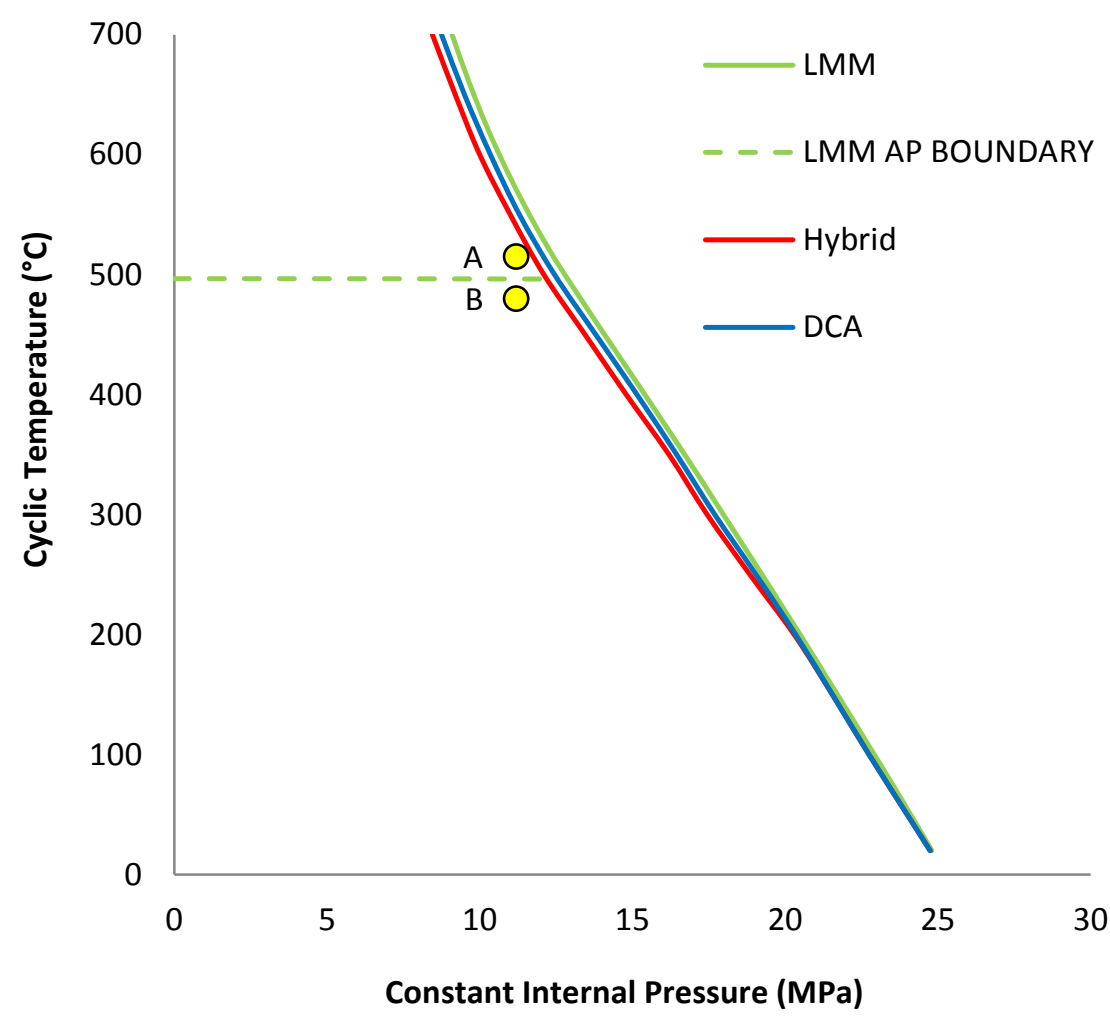

Fig. 4 - Load interaction plot for the Bree problem. 
Figure 5

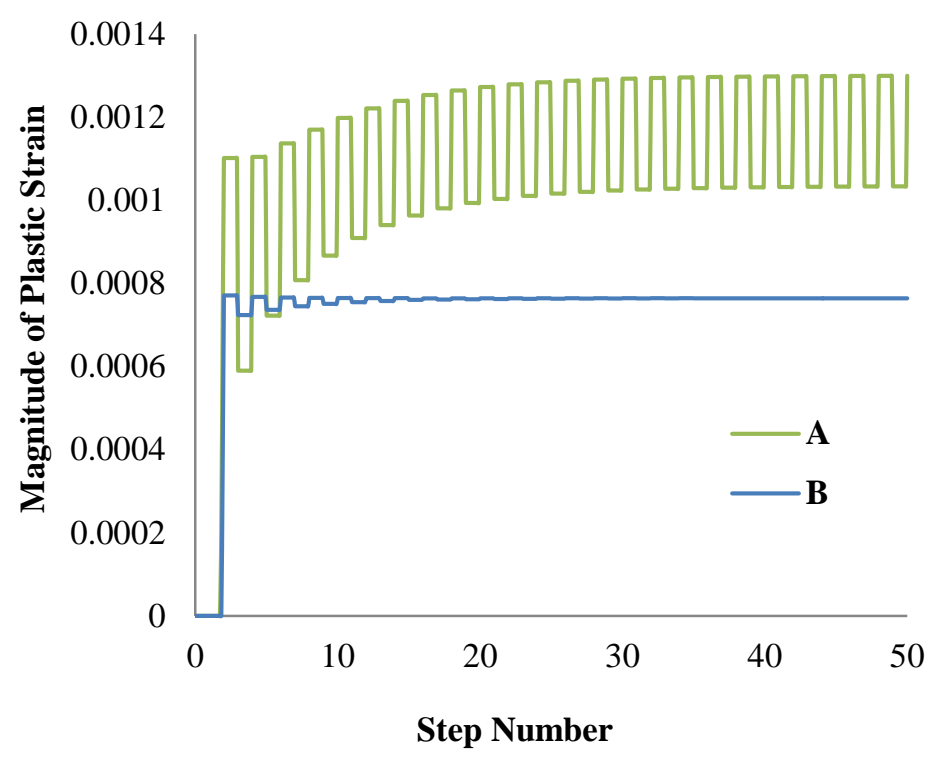

Fig. 5 - Step-by-step plastic strain results from points $A$ and $B$ respectively. 
Figure 6

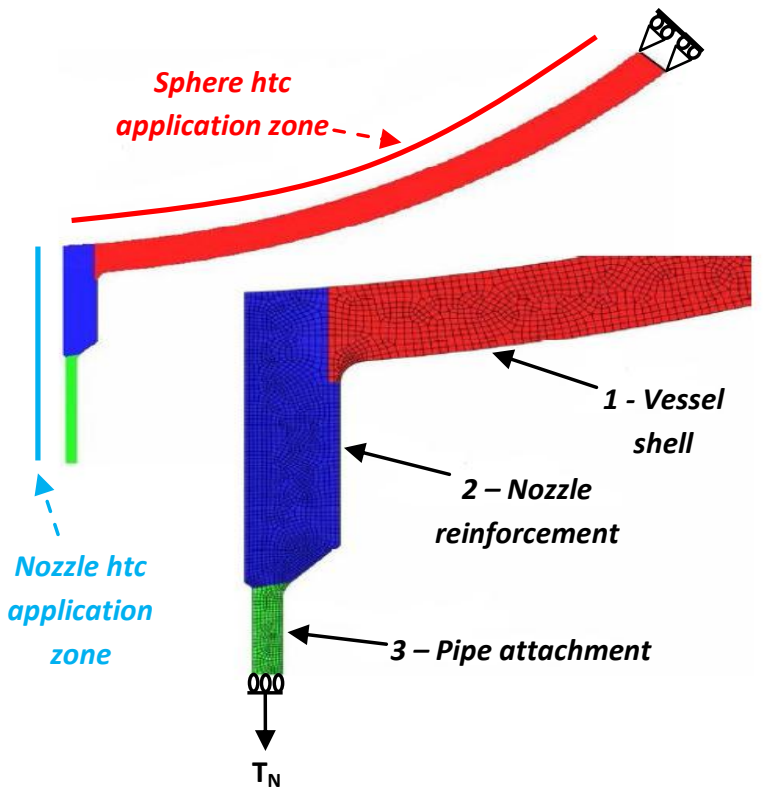

Fig. 6 - FE mesh, htc application zones and boundary conditions. 
Figure 7

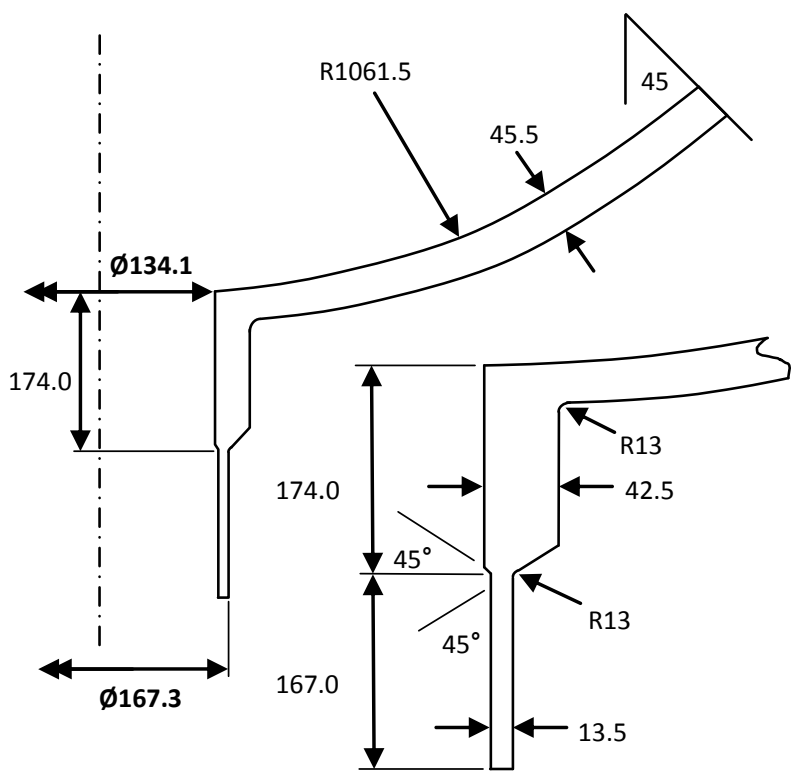

Fig. 7 - Dimensional data for the DBA problem. 


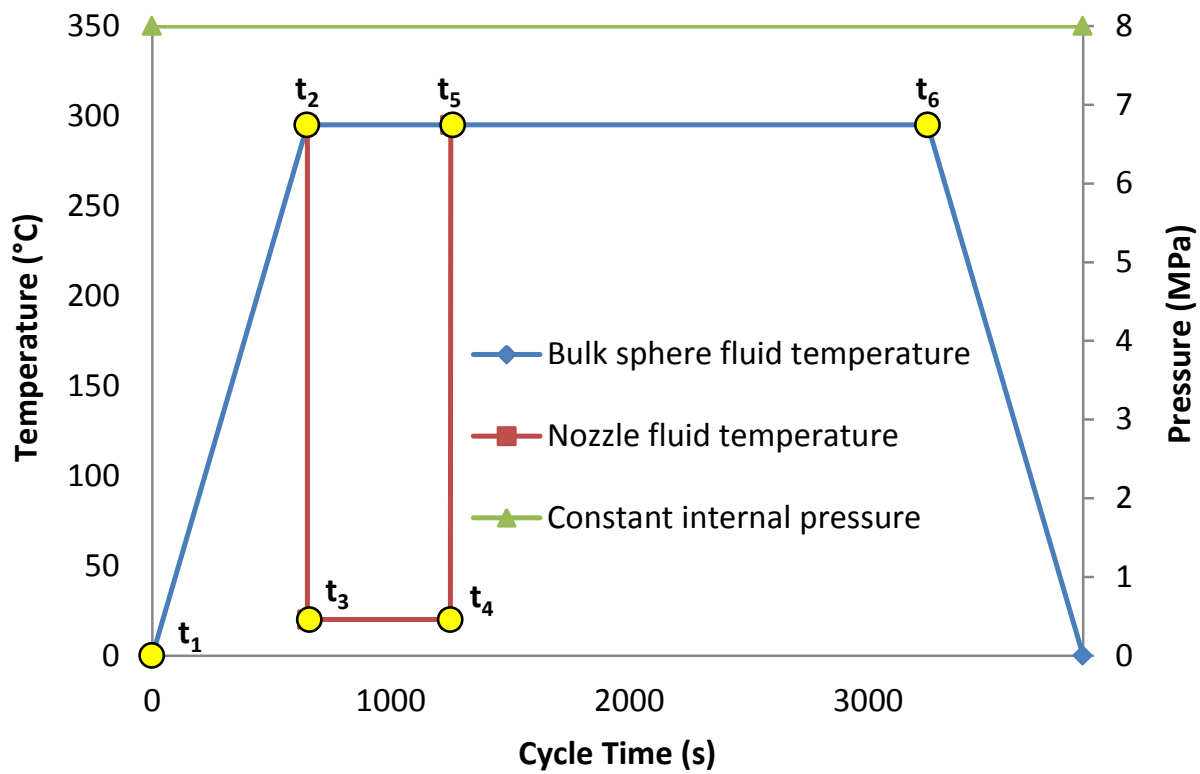

Fig. 8 - The thermo-mechanical operational load cycle. 
Figure 9

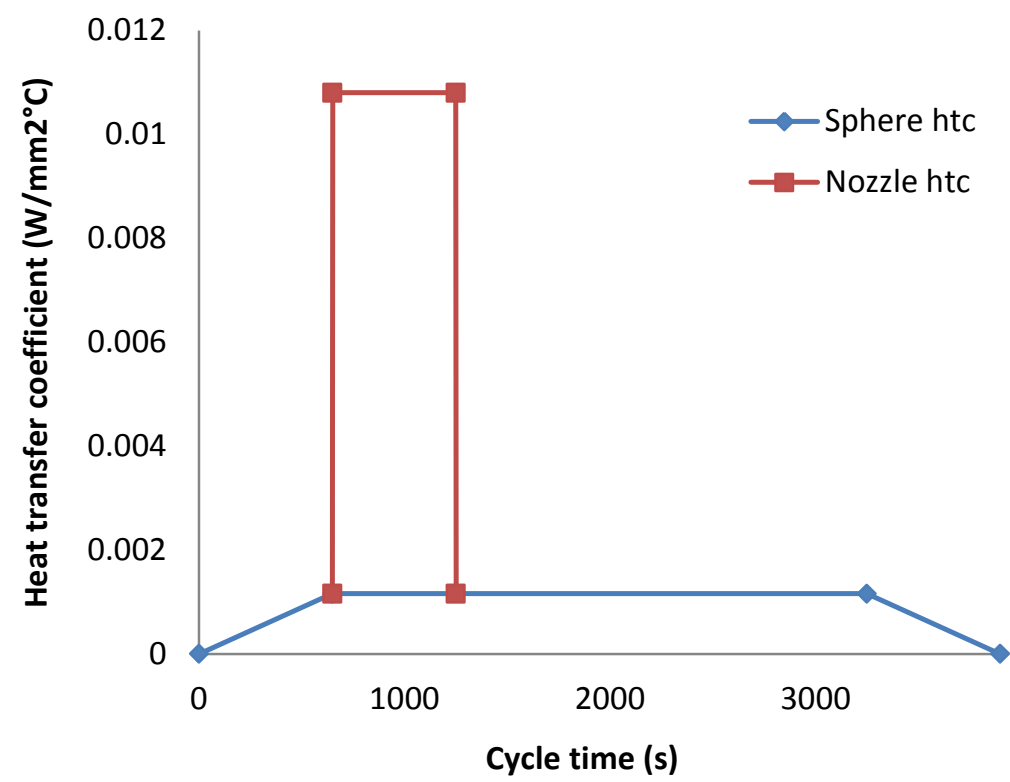

Fig. 9 - The htc application cycle. 


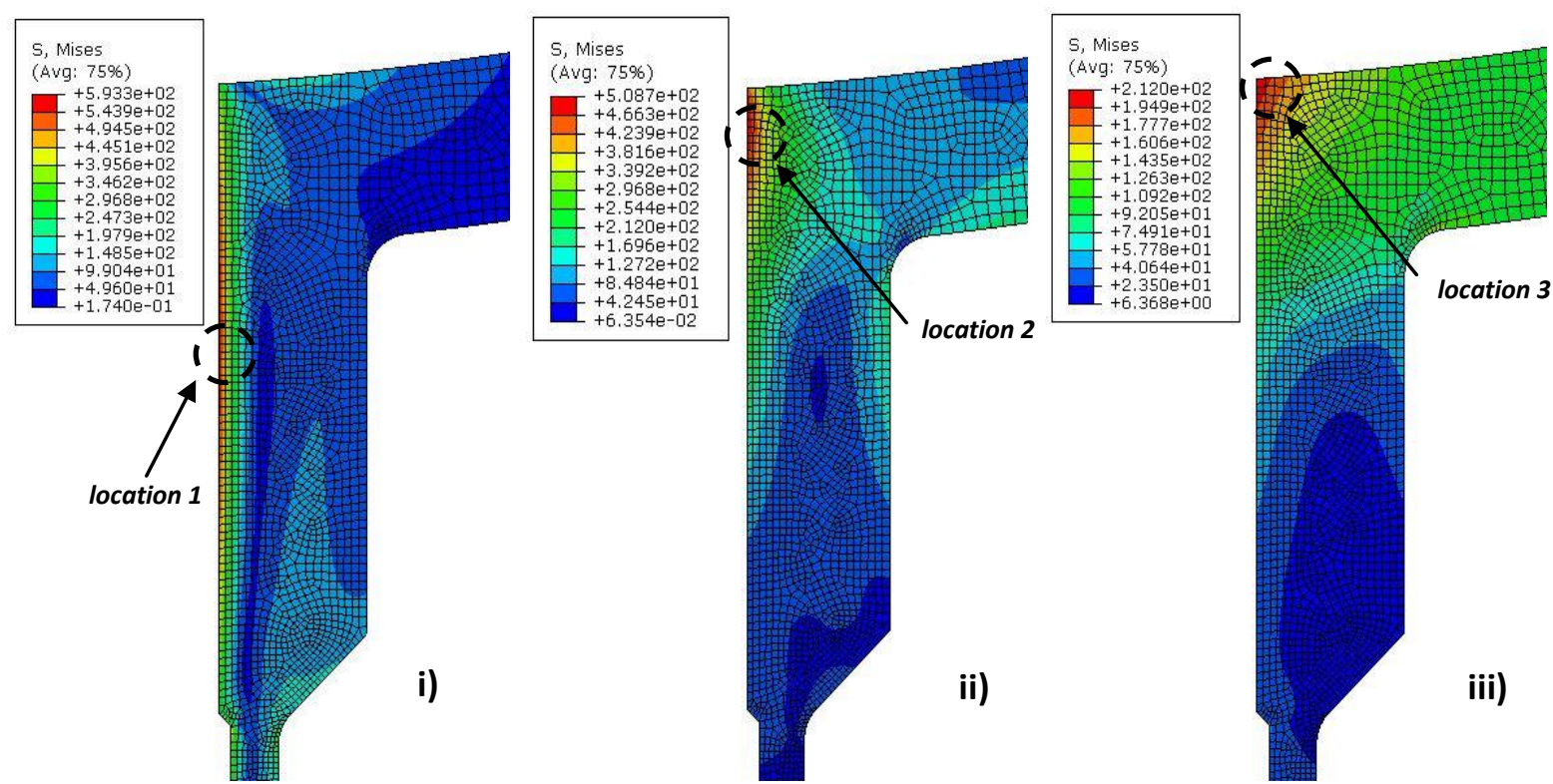

Figs. 10 i) The effective von Mises stress at $T_{c}+10 \mathrm{~s}$, with the peak value found at 'location 1 ', ii) The effective von Mises stress 600 s after a cold-media injection (i.e. at steady-state), with the peak value found at 'location 2 ' and iii) The effective von Mises stress due to the mechanical load component only, with the peak value found at 'Iocation 3'. 


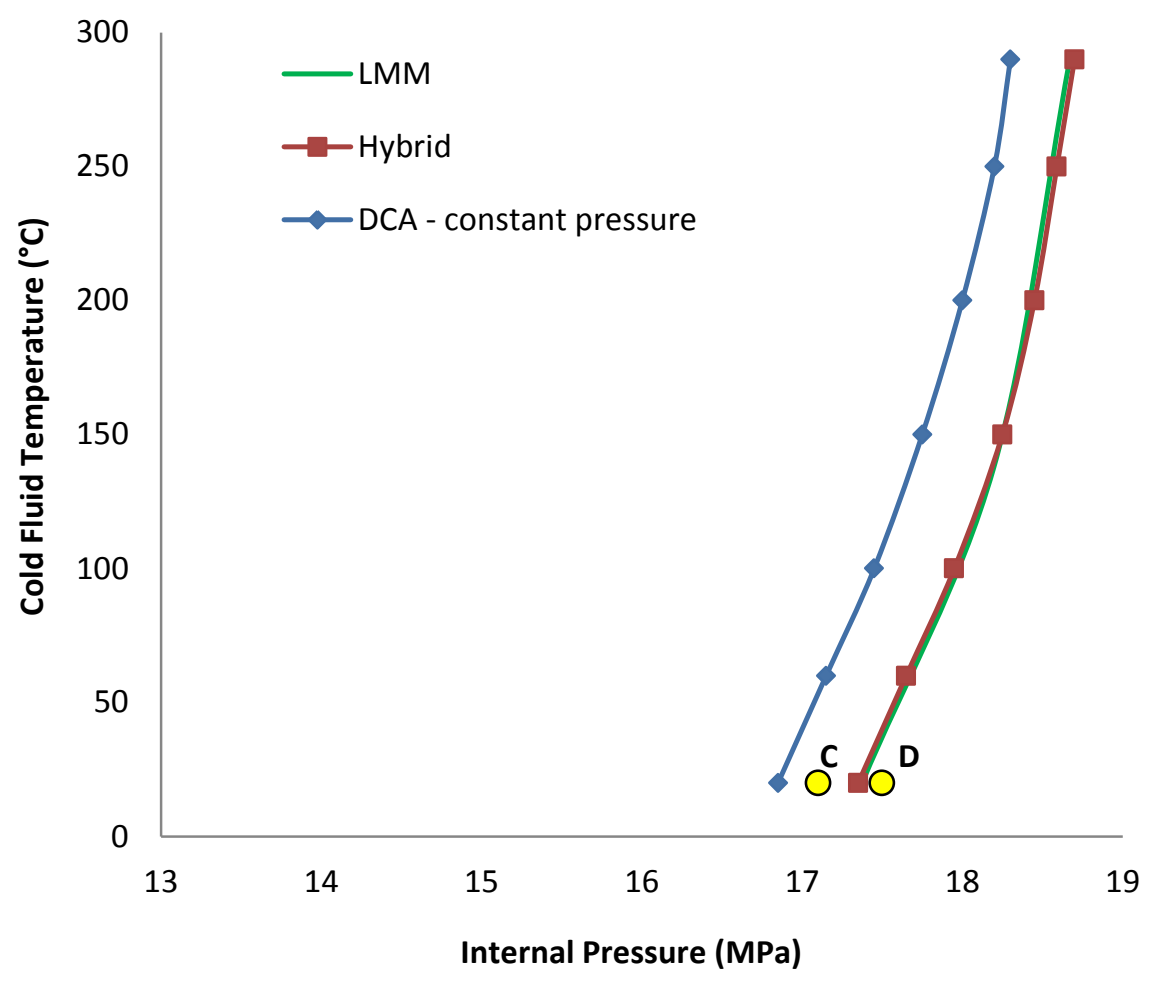

Fig. 11 - Comparison data obtained from the LMM, Hybrid and pure DCA methods [14]. 


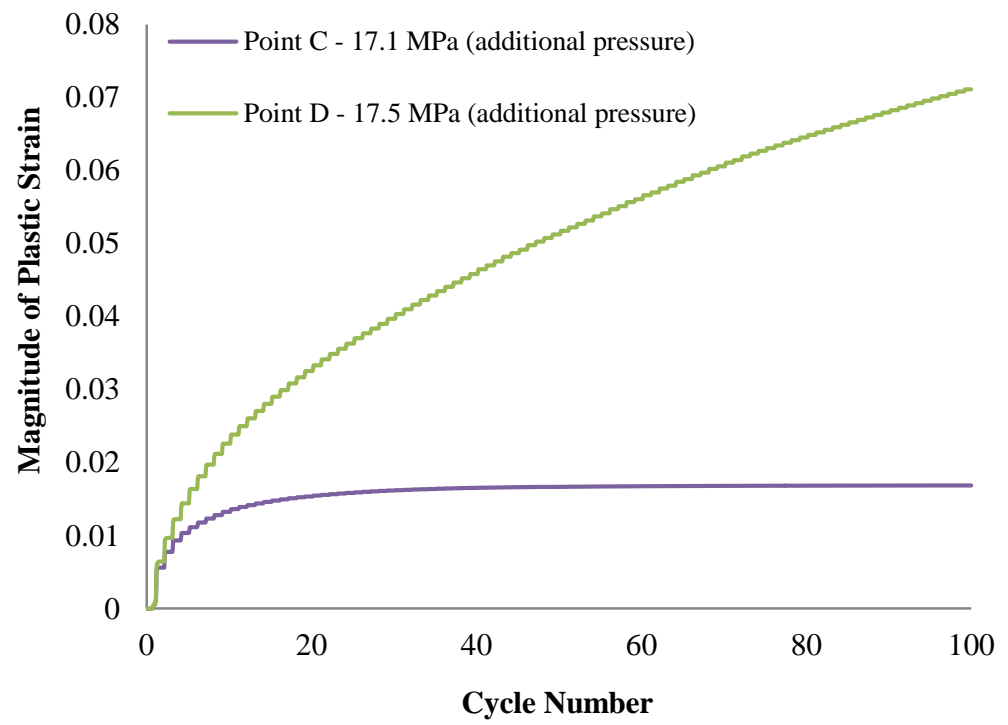

Fig. 12 - Step-by-step plastic strain results from points $C$ and $D$ respectively. 


$$
r
$$




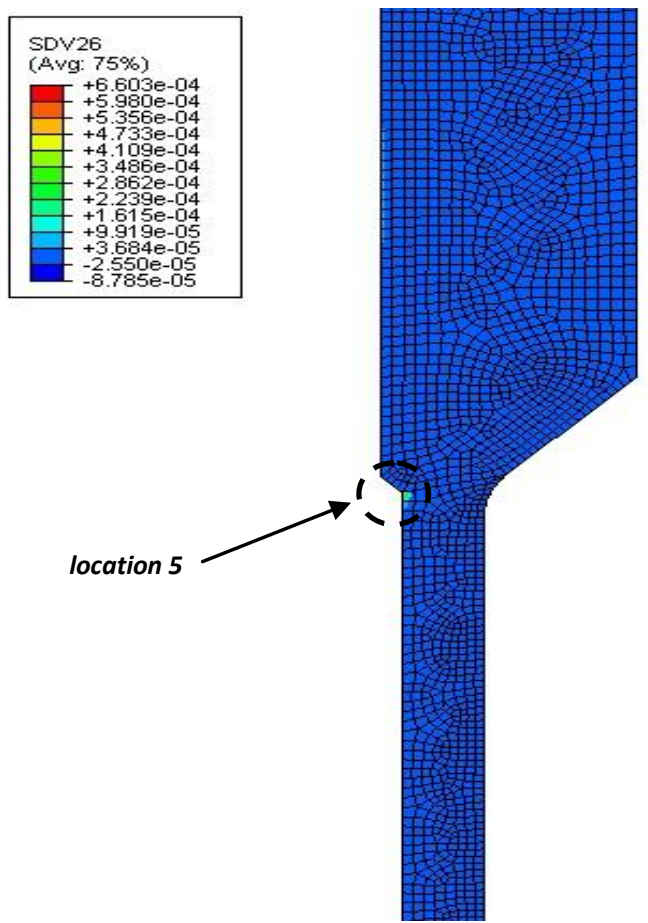

Fig. 14 - The maximum plastic strain range attributed to the cyclic thermal loading can be seen at 'location 5'. 


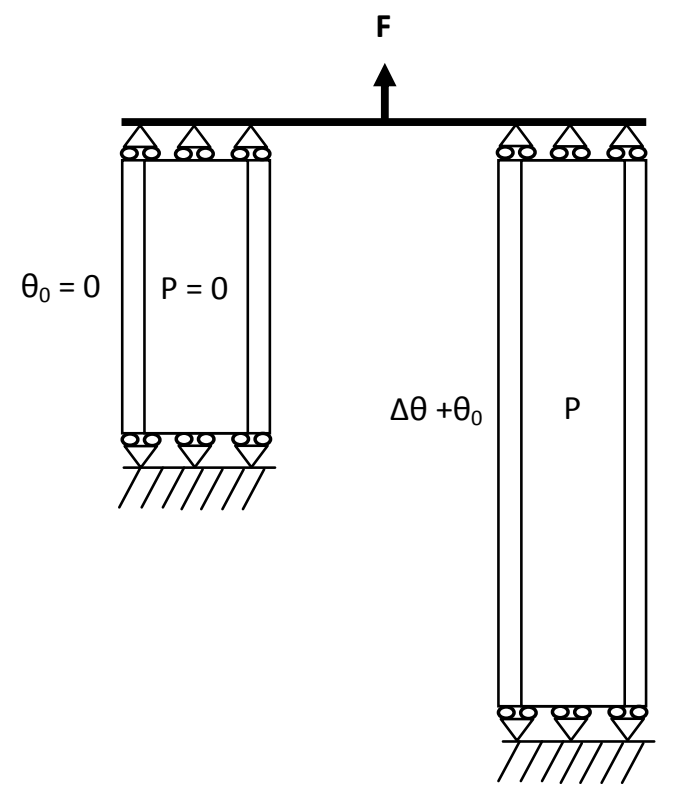

Fig. 15 - Loading and boundary conditions for the pressurised two-bar problem [14]. 


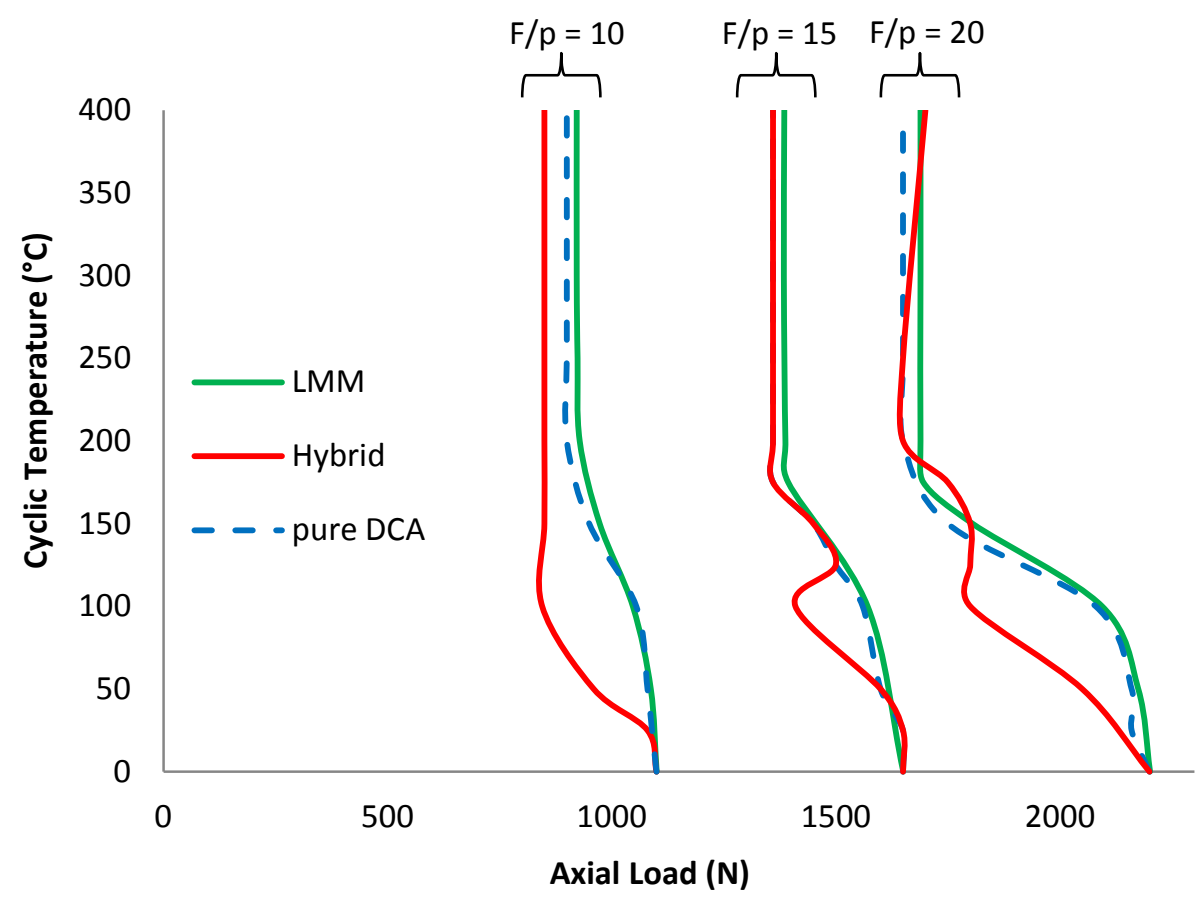

Fig. 16 - Ratchet limit interaction curves for various ratios of F/p. 